\title{
Differential gene expression analysis in glioblastoma cells and normal human brain cells based on GEO database
}

\author{
ANPING WANG and GUIBIN ZHANG \\ Department of Neurology, Xiangyang No. 1 People's Hospital, \\ Hubei University of Medicine, Xiangyang, Hubei 441000, P.R. China
}

Received July 10, 2017; Accepted September 6, 2017

DOI: $10.3892 / \mathrm{ol} .2017 .6922$

\begin{abstract}
The differentially expressed genes between glioblastoma (GBM) cells and normal human brain cells were investigated to performed pathway analysis and protein interaction network analysis for the differentially expressed genes. GSE12657 and GSE42656 gene chips, which contain gene expression profile of GBM were obtained from Gene Expression Omniub (GEO) database of National Center for Biotechnology Information (NCBI). The 'limma' data packet in 'R' software was used to analyze the differentially expressed genes in the two gene chips, and gene integration was performed using 'RobustRankAggreg' package. Finally, pheatmap software was used for heatmap analysis and Cytoscape, DAVID, STRING and KOBAS were used for protein-protein interaction, Gene Ontology (GO) and KEGG analyses. As results: i) 702 differentially expressed genes were identified in GSE12657, among those genes, 548 were significantly upregulated and 154 were significantly downregulated $(\mathrm{p}<0.01$, fold-change $>1$ ), and 1,854 differentially expressed genes were identified in GSE42656, among the genes, 1,068 were significantly upregulated and 786 were significantly downregulated $(\mathrm{p}<0.01$, fold-change $>1$ ). A total of 167 differentially expressed genes including 100 upregulated genes and 67 downregulated genes were identified after gene integration, and the genes showed significantly different expression levels in GBM compared with normal human brain cells $(\mathrm{p}<0.05)$. ii) Interactions between the protein products of 101 differentially expressed genes were identified using STRING and expression network was established. A key gene, called CALM3, was identified by Cytoscape software. iii) GO enrichment analysis showed that differentially expressed genes were mainly enriched in 'neurotransmitter:sodium symporter activity' and 'neurotransmitter transporter activity', which can affect the activity of
\end{abstract}

Correspondence to: Dr Guibin Zhang, Department of Neurology, Xiangyang No. 1 People's Hospital, Hubei University of Medicine, 15 Jiefang Road, Xiangyang, Hubei 441000, P.R. China

E-mail: g063ex@163.com

Key words: glioblastoma, differential expressed gene, GO enrichment, KEGG pathway analysis, protein interaction network neurotransmitter transportation. KEGG pathway analysis showed that the differentially expressed genes were mainly enriched in 'protein processing in endoplasmic reticulum', which can affect protein processing in endoplasmic reticulum. The results showed that: i) 167 differentially expressed genes were identified from two gene chips after integration; and ii) protein interaction network was established, and GO and KEGG pathway analyses were successfully performed to identify and annotate the key gene, which provide new insights for the studies on GBN at gene level.

\section{Introduction}

As the most malignant type of astrocytic tumors, the recurrence and mortality rates of GBM are extremely high (1). Studies have found that the molecular mechanisms of primary glioblastoma (GBM) and secondary GBM were different (2). Primary GBM is caused by the overexpression of epidermal growth factor receptor (EGFR), while secondary GBM is caused by the mutations of p53 (3). Due to the differential expression of a large number of genes in GBM, conventional biomolecular methods cannot be used to demonstrate the pathogenesis of GBM. Gene expression profile chip, which can measure the expression levels of a large number of genes, is an ideal approach for the analysis of molecular mechanism of GBM (4). In recent years, more and more gene expression profile data become available, and the use of bioinformatics to analyze gene expression profile data has become a new research hotspot (5). In this study, bioinformatics methods were used to analyze the data of gene expression profiles with an expectation of analyzing the differentially expressed genes between GBN and normal human brain cells, so as to provide new insights for the studies on the pathogenesis of GBM.

\section{Materials and methods}

Gene expression profile data. Data of gene chip GSE12657 and GSE42656 were obtained from GEO database. GSE12657 was from Neuropathology in the Department of Medicine at Imperial College London with 7 cases of GBM patients as experimental group and 5 cases of normal samples as a control group. GSE42656 was from Neuroscience and Trauma at Barts and the London School of Medicine and Dentistry with 5 cases of GBM patients as experimental group and 8 cases of 


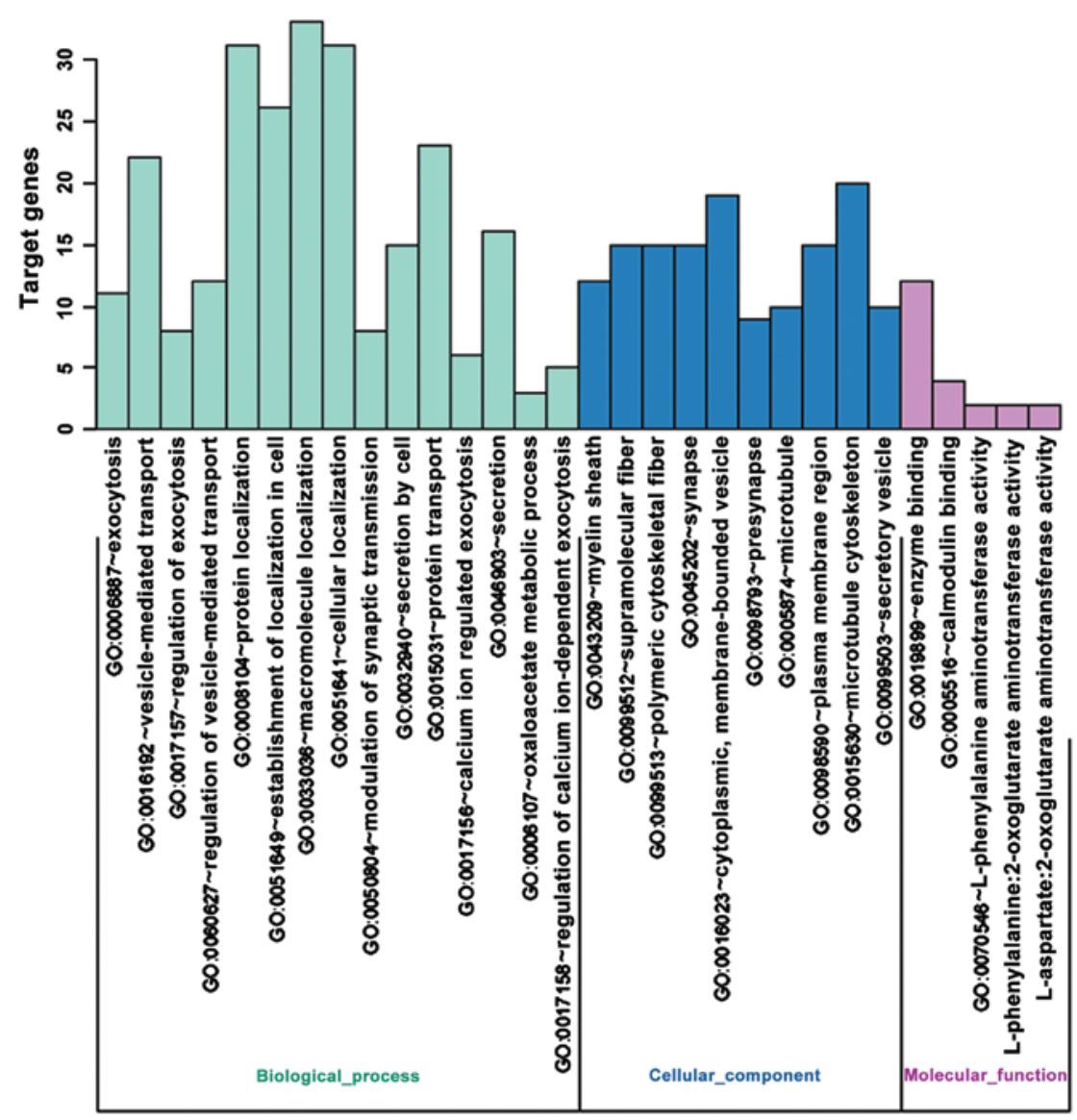

Figure 1. Results of GO enrichment. Abscissa is the enriched GO, and ordinate is the number and ratio of the differentially expressed genes. Different colors represent different GO classes, namely Molecular function, Biological process, and Cellular component. GO, Gene Ontology.

normal samples as a control group. This study was approved by the Ethics Committee of Xiangyang No. 1 People's Hospital, Hubei University of Medicine. Signed written informed consents were obtained from the patients and/or guardians.

Raw data preprocessing and screening and integration of differentially expressed genes. Affymetrix Expression Console and RMA algorithm were used for quality control, standardization and $\log 2$ conversion for the raw data of gene chips. Microarray data analysis package (Linear Models for Microarray Data, Limma) in ' $R$ ' software was used to screen the differentially expressed genes from raw data of two gene chips. Gene integration of differentially expressed genes identified from two gene chips was performed using RobustRankAggreg.

Gene Ontology $(G O)$ enrichment analysis. DAVID and the plug-in unit 'Bingo' of Cytoscape software (San Diego, CA, USA) were used for GO enrichment analysis and functional annotation after gene integration. Database for Annotation, Visualization and Integration Discovery (DAVID) analysis, DAVID network software (NIH, Bethesda, MD, USA) contains almost all major public bioinformatics resources. DAVID can be used to annotate gene-related biological mechanisms using standardized gene terminology.

DAVID knowledge base is designed to facilitate high-throughput gene functional analysis. DAVID provides a wide range of heterogeneous annotation data in a centralized location for a given gene list. DAVID enriches the biological information for individual genes. DAVID knowledge base can be downloaded from the following website: https://david.ncifcrf.gov/.

KEGG pathway analysis. KEGG pathway analysis and functional annotation for differentially expressed gene were performed using KOBAS 3.0 software (Peking University, Beijing, China).

KOBAS is the first software to use the hypergeometric distribution method to determine the significance of pathway enrichment. KOBAS has been successfully used in the study of different organisms such as plants, animals and bacteria. KOBAS server can be accessed at https://kobas.cbi.pku.edu.cn.

Protein interaction network analysis. STRING software (STRING 10.0; European Molecular Biology Laboratory, Heidelberg, Germany) was used to analyze the protein-protein interaction (PPI) of differentially expressed genes. PPI refers to the forming of protein complex by two or more protein molecules through non-covalent bonds. STRING can be accessed at https://string-db.org/.

\section{Results}

Screening of differentially expressed genes. A total of 702 differentially expressed genes were identified from gene chip GSE12657, and 548 genes were significantly upregulated and 154 genes were significantly downregulated $(\mathrm{p}<0.01$, fold-change $>1$ ). In gene chip GSE42656, 1,854 differentially 


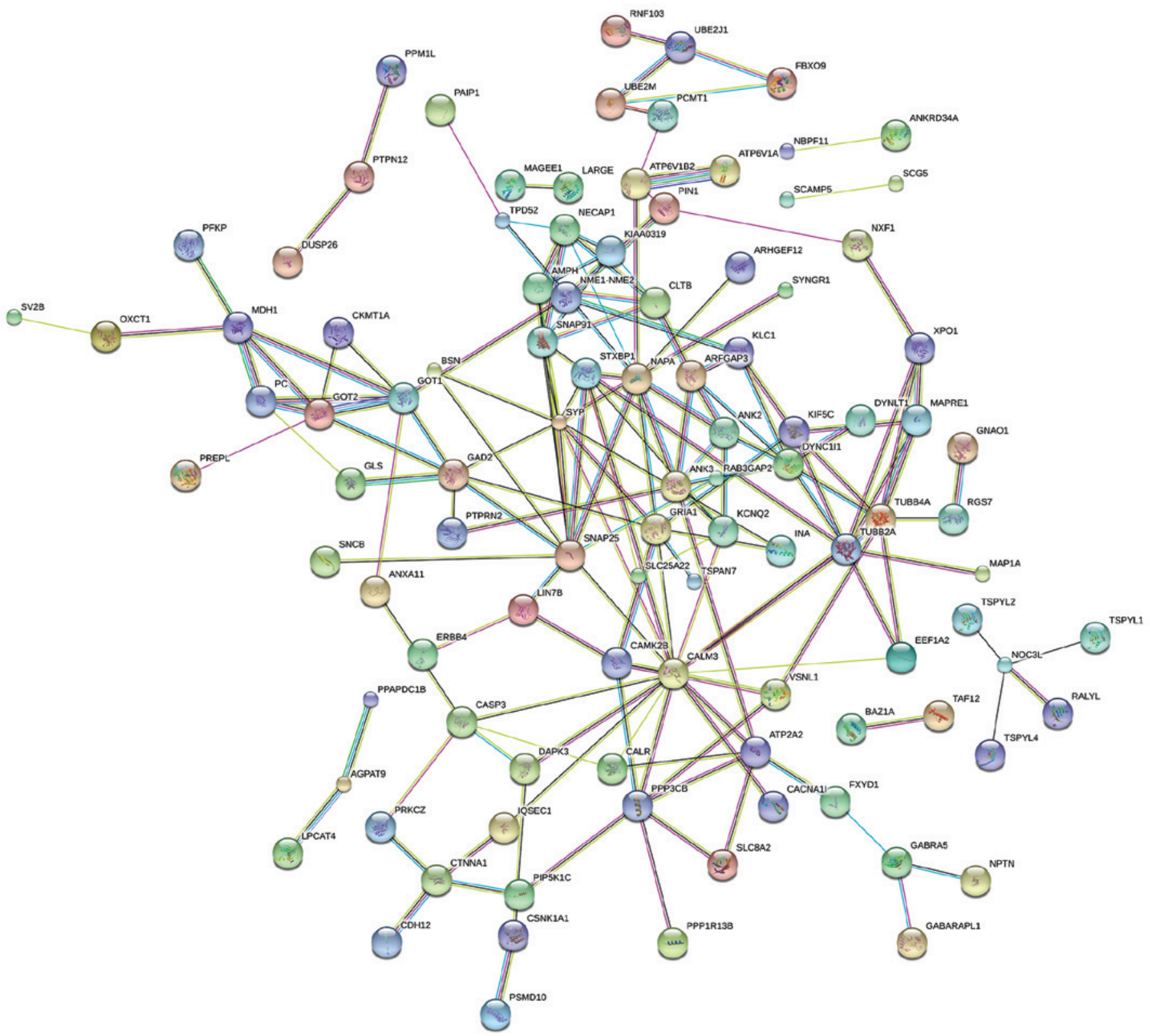

Figure 2. The diagram of protein interaction network. Circle represents the gene, and lines represent the protein interaction between the genes, and the information inside the circle describes protein structure: small nodes, protein of unknown 3D structure; large nodes, some 3D structure is known or predicted; a red line indicates the presence of fusion evidence; a green line, neighborhood evidence; a blue line, coocurrence evidence; a purple line, experimental evidence; a yellow line, text mining evidence; a light blue line, database evidence; a black line, coexpression evidence.

expressed genes were identified, and 1,068 genes were significantly upregulated and 786 genes were significantly downregulated $(\mathrm{p}<0.01$, fold-change $>1)$. After gene integration, 167 differentially expressed genes including 67 downregulated genes and 100 upregulated genes were identified. Those genes showed significantly different expression levels in GBM compared with normal human brain cells $(\mathrm{p}<0.05)$.

GO enrichment analysis. The list of differentially expressed genes was submitted to DAVID Bioinformatics Resource Network (https://david.ncifcrf.gov/) with OFFICIAL-GENE-SYMBOL and Gene List were selected. All other parameters were default.

Differentially expressed genes were mainly enrich in 'neurotransmitter:sodium symporter activity' and 'neurotrans- mitter transporter activity', which can affect the activity of neurotransmitter transportation (Fig. 1).

KEGG pathway analysis. KEGG pathway analysis and functional annotation were performed using KOBAS 3.0 software. Four key KEGG pathways included: 'Dopaminergic synapses', 'MAPK signaling pathway', 'Glyoxylate and dicarboxylate metabolism' and 'Protein processing in endoplasmic reticulum' (Table I).

Protein interaction network analysis. Thirty outstanding proteins were identified through PPI analysis of STRING software. SNAP25, SYP, NAPA, TUBB2A and TUBB4A proteins were relatively more important. As the most important protein, CALM3 connected 17 nodes (Figs. 2 and 3). 
Table I. Results of KEGG pathway analysis.

\begin{tabular}{llll}
\hline Term & Count & P-value & FDR \\
\hline hsa04141:Protein processing in endoplasmic reticulum & 3 & 0.000309962 & 0.01239848 \\
hsa04728:Dopaminergic synapse & 2 & 0.004768424 & 0.095368478 \\
hsa04010:MAPK signaling pathway & 2 & 0.017081905 & 0.138117097 \\
hsa00630:Glyoxylate and dicarboxylate metabolism & 1 & 0.022356127 & 0.138117097 \\
hsa03410:Base excision repair & 1 & 0.026161426 & 0.138117097 \\
hsa04130:SNARE interactions in vesicular transport & 1 & 0.026920764 & 0.138117097 \\
hsa04962: Vasopressin-regulated water reabsorption & 1 & 0.034482696 & 0.138117097 \\
hsa03420:Nucleotide excision repair & 1 & 0.036740165 & 0.138117097 \\
hsa05030:Cocaine addiction & 1 & 0.038242305 & 0.138117097 \\
hsa04978: Mineral absorption & 1 & 0.040491267 & 0.138117097
\end{tabular}

Term, enriched KEGG; count, the number of differentially expressed genes of each term; P-value, enrichment statistical P-value; FDR, P-value after correction.

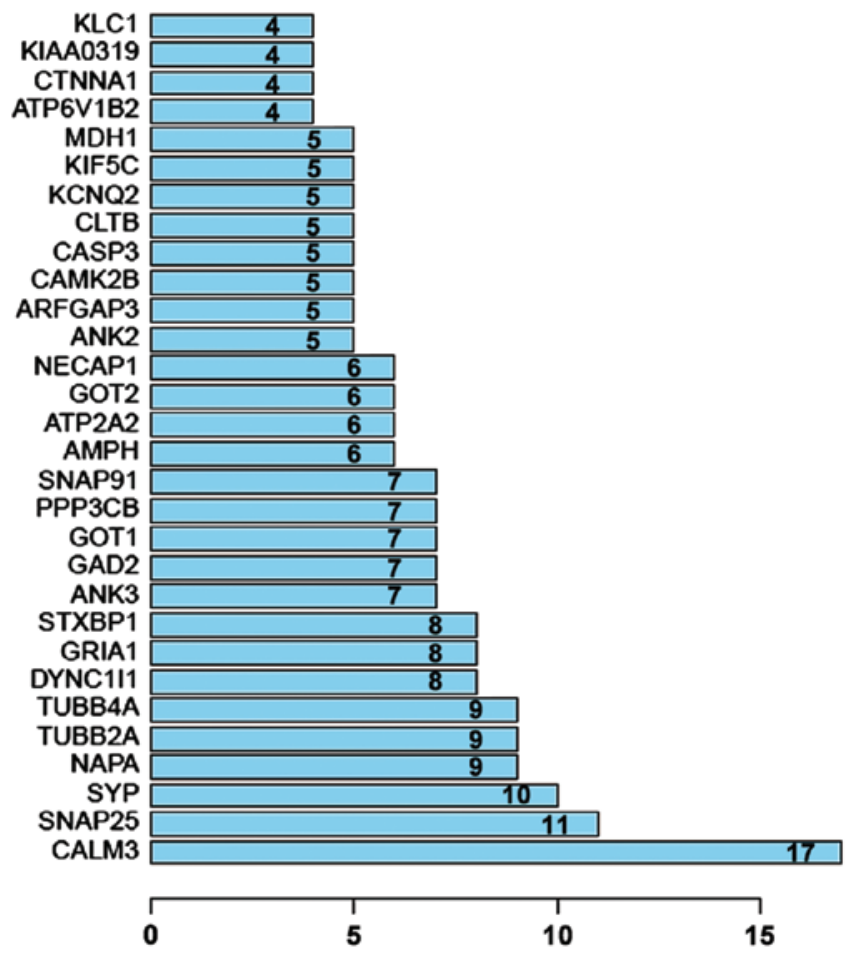

Figure 3. Core protein histogram. The vertical coordinates is the gene name, and the horizontal coordinates is the number of adjacent genes, and the height represents the number of lines connected genes.

\section{Discussion}

As the most common and most aggressive diffuse glioma (6), GBM is the most malignant type of astrocytic tumor. GBM develops from cortex and shows infiltrative development. GBM can simultaneously affect several lobes (7). GBM is characterized by heterogeneity of morphology, genetics and gene expression (8). In recent years, with the rapid development of bioinformatics, the use of gene expression profiles to explore the relationship between gene differential expression and disease development has attracted more and more attention. However, those data have not been comprehensively investigated (9). In this study, gene chip data were used to identify the differentially expression genes between GBM and normal human brain cells through enrichment analysis and protein interaction analysis with the expectation of exploring the pathogenesis of GBM. As the most common endogenous primary brain tumor in adults, GBM represents the most common type of diffuse glioma (10). Central Brain Tumor Registry of the United States (CBTRUS) reported that GBM mainly affect patients between 75 and 84 years, and the incidence of GBM showing an increasing trend and incidence in white males is ever higher (11). GBM is rare in children and only account for $\sim 3 \%$ of all primary brain and CNS tumors. The five-year survival rate is about $12 \%$ for children and less than $5 \%$ for adults (12). In spite of the achievement in the treatment of GBM, the survival rate of patients is still very low (13). GBM exists in cerebral cortex and showed strong invasion ability, so the course of disease is short and average survival period is only $\sim 14$ months (14). Without treatment, GBM patients cannot survive more than 2 months, so the development of effective diagnosis and treatment methods is always needed (15). Previous studies have shown that the development of GBM is very complex and is related to the abnormal expression of proto-oncogenes or tumor suppressor genes, which can lead to abnormal activation or dysregulation of intracellular signaling pathways (16).

In this study, GEO public database was used. The 'limma' package was used to analyze and integrate data. A total of 167 differentially expressed genes were identified. GO enrichment analysis and KEGG pathway analysis showed that the differentially expressed genes were mainly involved in neurotransmitter transporter activity, neurotransmitter:sodium symporter activity, cellular processes, solute: sodium symporter activity, monoplast processes, protein processing in endoplasmic reticulum, dopaminergic synapses, MAPK signal transduction pathway and glyoxylate and dicarboxylate metabolism. Neurotransmitter transporter activity and protein processing in endoplasmic reticulum are the two most outstanding pathways. However, CALM3 protein is the most influential protein on GBM in the network formed by CALM3, SNAP25, SYP, NAPA, TUBB2A, TUBB4A, DYNC1I1, 
GRIA1, STXBP1, ANK3, GOT1, GAD2, PPP3CB, SNAP91, AMPH, ATP2A2 and other genes. CALM3 gene was reported to be closely associated with long QT syndrome (17). More studies are needed to investigate the mechanism of the roles of CALM3.

Bioinformatics is a new discipline that combines biological science and computer science (18). Major bioinformatics tools were used in this study to identify the differentially expressed genes. Public database of gene chip data was used in this study, which significantly reduced the use of financial and material resources. Based on the strict inclusion criteria, the most reliable gene chip data were selected to avoid errors.

This study is limited by the small sample size. Gene expression in GBM can be altered by certain factors (19), and the small sample size failed to cover different races and regions, which can affected the gene expression in GBM (20).

In this study, CALM3 gene was proved to be related to the protein processing and transporter activity in GBM. Our future study will focus on those pathways. Studies on GBM at gene level are rare. Therefore, more studies are needed to improve the diagnosis, treatment and prognosis of GBM.

\section{References}

1. Lathia JD, Mack SC, Mulkearns-Hubert EE, Valentim CL and Rich JN: Cancer stem cells in glioblastoma. Genes Dev 29: 1203-1217, 2015

2. Jin Z, Jin RH, Ma C, Li HS and Xu HY: Serum expression level of miR-504 can differentiate between glioblastoma multiforme and solitary brain metastasis of non-small cell lung carcinoma. J BUON 22: 474-480, 2017.

3. Sharma V, Dixit D, Koul N, Mehta VS and Sen E: Ras regulates interleukin-1 $\beta$-induced HIF- $1 \alpha$ transcriptional activity in glioblastoma. J Mol Med (Berl) 89: 123-136, 2011.

4. Reed GJ, Boczek NJ, Etheridge SP and Ackerman MJ: CALM3 mutation associated with long QT syndrome. Heart Rhythm 12: 419-422, 2015.

5. Olar A and Aldape KD: Using the molecular classification of glioblastoma to inform personalized treatment. J Pathol 232: 165-177, 2014.

6. Lun M, Lok E, Gautam S, Wu E and Wong ET: The natural history of extracranial metastasis from glioblastoma multiforme. J Neurooncol 105: 261-273, 2011.

7. Goodenberger ML and Jenkins RB: Genetics of adult glioma. Cancer Genet 205: 613-621, 2012.

8. Jones TS and Holland EC: Standard of care therapy for malignant glioma and its effect on tumor and stromal cells. Oncogene 31 : 1995-2006, 2012.
9. Stupp R, Hegi ME, Mason WP, van den Bent MJ, Taphoorn MJ, Janzer RC, Ludwin SK, Allgeier A, Fisher B, Belanger K, et al; European Organisation for Research and Treatment of Cancer Brain Tumour and Radiation Oncology Groups; National Cancer Institute of Canada Clinical Trials Group: Effects of radiotherapy with concomitant and adjuvant temozolomide versus radiotherapy alone on survival in glioblastoma in a randomised phase III study: Five-year analysis of the EORTC-NCIC trial. Lancet Oncol 10: 459-466, 2009.

10. Bo LJ, Wei B, Li ZH, Wang ZF, Gao Z and Miao Z: Bioinformatics analysis of miRNA expression profile between primary and recurrent glioblastoma. Eur Rev Med Pharmacol Sci 19: 3579-3586, 2015

11. Carro MS, Lim WK, Alvarez MJ, Bollo RJ, Zhao X, Snyder EY, Sulman EP, Anne SL, Doetsch F, Colman H, et al: The transcriptional network for mesenchymal transformation of brain tumours. Nature 463: 318-325, 2010.

12. Lathia JD, Gallagher J, Myers JT, Li M, Vasanji A, McLendon RE, Hjelmeland AB, Huang AY and Rich JN: Direct in vivo evidence for tumor propagation by glioblastoma cancer stem cells. PLoS One 6: e24807, 2011.

13. Xie Q, Mittal S and Berens ME: Targeting adaptive glioblastoma: An overview of proliferation and invasion. Neuro-oncol 16: 1575-1584, 2014

14. Batchelor TT, Reardon DA, de Groot JF, Wick W and Weller M: Antiangiogenic therapy for glioblastoma: Current status and future prospects. Clin Cancer Res 20: 5612-5619, 2014.

15. Xie Y, Bergström T, Jiang Y, Johansson P, Marinescu VD Lindberg N, Segerman A, Wicher G, Niklasson M, Baskaran S, et al: The human glioblastoma cell culture resource: Validated cell models representing all molecular subtypes. EBioMedicine 2: 1351-1363, 2015.

16. Guo E, Wang Z and Wang S: miR-200c and miR-141 inhibit ZEB1 synergistically and suppress glioma cell growth and migration. Eur Rev Med Pharmacol Sci 20: 3385-3391, 2016.

17. Joo KM, Kim J, Jin J, Kim M, Seol HJ, Muradov J, Yang H, Choi YL, Park WY, Kong DS, et al: Patient-specific orthotopic glioblastoma xenograft models recapitulate the histopathology and biology of human glioblastomas in situ. Cell Rep 3: 260-273, 2013.

18. Meyer M, Reimand J, Lan X, Head R, Zhu X, Kushida M, Bayani J, Pressey JC, Lionel AC, Clarke ID, et al: Single cell-derived clonal analysis of human glioblastoma links functional and genomic heterogeneity. Proc Natl Acad Sci USA 112: 851-856, 2015.

19. Ogden AT, Waziri AE, Lochhead RA, Fusco D, Lopez K, Ellis JA, Kang J, Assanah M, McKhann GM, Sisti MB, et al: Identification of $\mathrm{A} 2 \mathrm{~B}^{+} \mathrm{CD} 133^{-}$tumor-initiating cells in adult human gliomas. Neurosurgery 62: 505-515, 2008.

20. Savary K, Caglayan D, Caja L, Tzavlaki K, Bin Nayeem S, Bergström T, Jiang Y, Uhrbom L, Forsberg-Nilsson K, Westermark B, et al: Snail depletes the tumorigenic potential of glioblastoma. Oncogene 32: 5409-5420, 2013. 